EPJ Web of Conferences 43, 03005 (2013)

DOI: $10.1051 /$ epjconf/20134303005

(C) Owned by the authors, published by EDP Sciences, 2013

\title{
Combined photometric and asteroseismic constraints on the properties of NGC 6791
}

\author{
D. Bossini ${ }^{1,2, a}$, L. Girardi ${ }^{3}$, A. Miglio ${ }^{2}$, A. Bressan ${ }^{4}$, \\ P. Marigo ${ }^{1}$ and members of KASC WG2 \\ ${ }^{1}$ Dipartimento di Fisica e Astronomia, Università di Padova, Vicolo dell'Osservatorio 2, \\ 35122 Padova, Italy \\ ${ }^{2}$ School of Physics and Astronomy, University of Birmingham, Edgbaston, Birmingham B15 \\ 2TT, UK \\ 3 Osservatorio Astronomico di Padova - INAF, Vicolo dell'Osservatorio 5, 35122 Padova, Italy \\ ${ }^{4}$ SISSA, via Bonomea 265, 34136 Trieste, Italy
}

\begin{abstract}
We compare a grid of isochrones with observational constraints on NGC 6791 considering photometric and asteroseismic data, both together and separately. We apply a method, based on Bayesian statistics, to identify the best-fit solutions and the uncertainties on the estimated global properties of the cluster.
\end{abstract}

\section{INTRODUCTION}

Thanks to the recent developments of observational techniques in asteroseismology, solar-like oscillations were detected in giant stars in the open clusters NGC 6791 and NGC 6819 from the light curves provided by the NASA's Kepler satellite [1]. Using these new data, Basu et al. [2] derived the average mass of the stars in the red giant branch (RGB) for both clusters, as well as an estimate of their age. In our work, we determine the properties of NGC 6791 combining the photometric [3] and asteroseismic data [4]. We use stellar models computed with the PAdova-tRieste Stellar Evolution Code (PARSEC, [5]). Through numerical methods based on isochrone-fitting techniques, we estimate the global properties of the cluster, like metallicity $(Z)$, initial helium mass fraction $(Y)$, and age, using photometric and asteroseismic data separately, finding the best-fits models for both of them. Our purpose is to estimate more precisely and self-consistently the uncertainties on all cluster parameters, joining the results within the corresponding errors bars.

\section{OUR METHOD}

Our method consists in a sequence of steps. First of all, we compute a grid of isochrones, with the ranges and steps of the parameters shown in Table 1. Second, we compare each isochrone from the grid with the observed photometric data, measuring the goodness of the fit (using the likelihood function, see e.g. [6]). Based on the likelihood, we find the best-fit and finally we identify the set of isochrones within $1 \sigma$ from the best fit. In photometry, we fit the isochrones in the Magnitude-Magnitude Diagram (MMD) using V and B magnitudes (Fig. 1). In asteroseismology, we fit only RGB stars

\footnotetext{
ae-mail: dbossini@bison.ph.bham.ac.uk
} 


\section{EPJ Web of Conferences}

Table 1. Parameters of the isochrone grids used in this work.

\begin{tabular}{|l|c|c|c|}
\hline Parameter & Min & Max & Step \\
\hline$Z$ & 0.030 & 0.050 & 0.005 \\
\hline$Y$ & 0.28 & 0.36 & 0.02 \\
\hline Log(age/yr) & 9.8 & 10 & 0.005 \\
\hline$E_{B-V}$ & 0.04 & 0.18 & 0.01 \\
\hline$(m-M)_{0}$ & 12.80 & 13.26 & 0.02 \\
\hline
\end{tabular}

Figure 1. CMD showing isochrones within 1- $\sigma$ of the best-fit model obtained using photometric constraints.

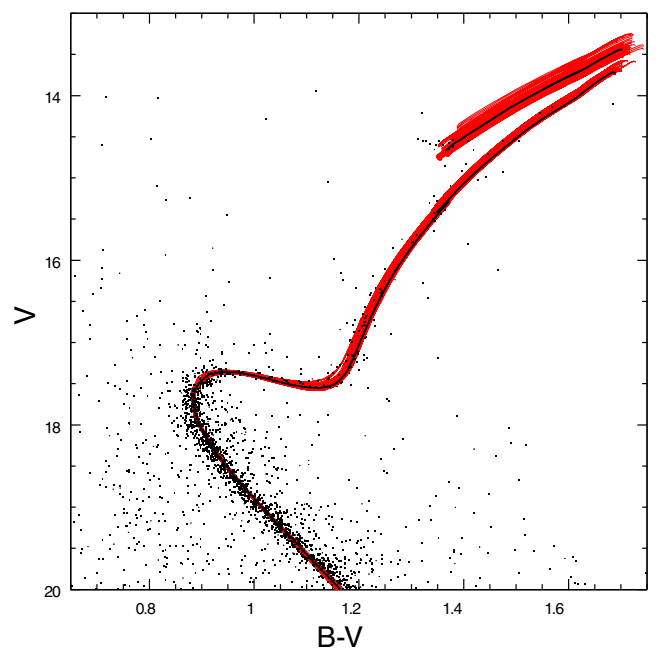

Figure 2. Isochrones within $1-\sigma$ of the best-fit model obtained using asteroseismic constraints shown on a $v_{\max } 0.75 / \Delta \nu-v_{\max }$ diagram.

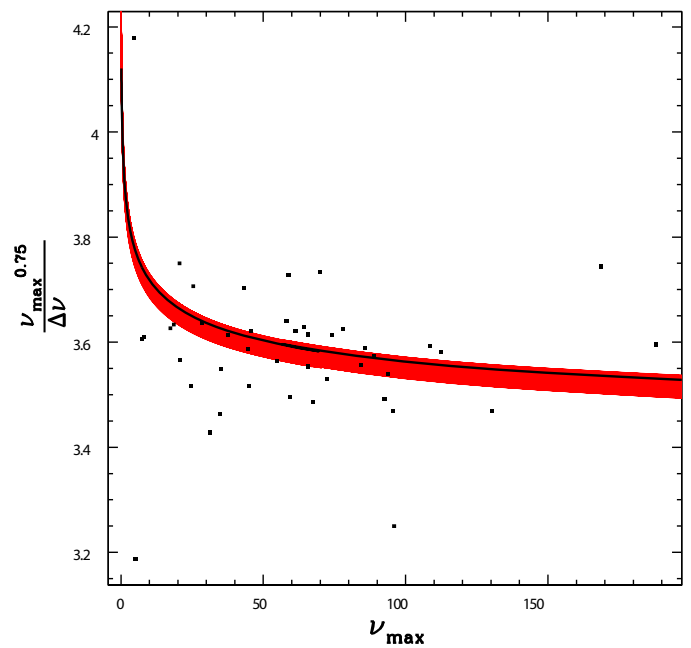

on the variables $\Delta v$ and $v_{\max }$ which, in the isochrones, are estimated from $L, M$ and $T_{\text {eff }}$ using scaling relations (see e.g. [7]). Results of the fit using asteroseismic constraints only is presented in Fig. 2.

At this point, we have two different best solutions and acceptable ranges of parameters for NGC 6791. In order to find the best solution taking into account both photometric and seismic constraints, we combine the likelihood from the two fits (Table 2). 
Ageing Low Mass Stars: From Red Giants to White Dwarfs

Table 2. Summary of the results obtained combining photometric and asteroseismic constraints.

\begin{tabular}{lccc}
\hline parameter & lower limit & best-fit & upper limit \\
\hline$Z$ & 0.036 (or less) & 0.042 & $>0.05$ \\
$Y$ & $<0.28$ (or less) & 0.28 & 0.32 \\
$\log ($ age/yr) & $9.895(7.85 \mathrm{Gyr})$ & $9.93(8.50 \mathrm{Gyr})$ & $9.97(9.30 \mathrm{Gyr})$ \\
$\log ($ age/yr) & 9.895 & 9.930 & 9.970 \\
$\bar{M}_{\mathrm{RGB}}\left(M_{\odot}\right)$ & 1.18 & 1.21 & 1.25 \\
\hline
\end{tabular}

\section{DISCUSSION AND FUTURE PROSPECTIVE}

We presented our method to determine global properties of open clusters, combining photometric and asteroseismic constraints. We also showed our preliminary results on NGC 6791. Unsurprisingly, the range of mass of giants on the RGB (Table 2) is compatible with the mass estimated by [2] and [8], $\bar{M}_{\mathrm{RGB}}=1.23 \pm 0.02 M_{\odot}$. The final results will be presented in Bossini et al. (in prep) where an improved version our method will be presented, spectroscopic constraints will be taken into account, as well as seismic constraints beyond simple scaling relations.

\section{References}

[1] R.L. Gilliland, T.M. Brown, J. Christensen-Dalsgaard, H. Kjeldsen, C. Aerts, T. Appourchaux, S. Basu, T.R. Bedding, W.J. Chaplin, M.S. Cunha et al., PASP 122, 131 (2010)

[2] S. Basu, F. Grundahl, D. Stello, T. Kallinger, S. Hekker, B. Mosser, R.A. García, S. Mathur, et al., ApJ 729, L10 (2011)

[3] P.B. Stetson, H. Bruntt, F. Grundahl, PASP 115, 413 (2003)

[4] D. Stello, D. Huber, T. Kallinger, S. Basu, B. Mosser, S. Hekker, S. Mathur, R.A. García, T.R. Bedding, H. Kjeldsen et al., ApJ 737, L10 (2011)

[5] A. Bressan, P. Marigo, L. Girardi, B. Salasnich, C. Dal Cero, S. Rubele, A. Nanni, MNRAS 427, 127 (2012)

[6] E. Tolstoy, A. Saha, ApJ 462, 672 (1996)

[7] H. Kjeldsen, T.R. Bedding, A\&A 293, 87 (1995)

[8] A. Miglio, K. Brogaard, D. Stello, W.J. Chaplin, et al., MNRAS 419, 2077 (2012) 